\title{
A Cotton (Gossypium hirsutum) Myo-Inositol-1-Phosphate Synthase (GhMIPS1D) Gene Promotes Root Cell Elongation in Arabidopsis
}

\author{
Rendi Ma ${ }^{1,+}$, Wangyang Song ${ }^{1,+}$, Fei Wang ${ }^{1,+}{ }^{+}$, Aiping Cao ${ }^{1}$, Shuangquan Xie ${ }^{1}$, Xifeng Chen ${ }^{1}$, \\ Xiang Jin ${ }^{1,2, *}$ and Hongbin $\mathrm{Li}^{1, *(1)}$ \\ 1 College of Life Sciences, Key Laboratory of Xinjiang Phytomedicine Resource and Utilization of Ministry of \\ Education, Shihezi University, Shihezi 832003, China; mrendi118@163.com (R.M.); \\ swywinner@163.com (W.S.); wangfshzu@163.com (F.W.); aiping9smile@sina.com (A.C.); \\ xiesq@shzu.edu.cn (S.X.); cxf_cc@shzu.edu.cn (X.C.) \\ 2 Ministry of Education Key Laboratory for Ecology of Tropical Islands, College of Life Sciences, \\ Hainan Normal University, Haikou 571158, China \\ * Correspondence: jinx@hainnu.edu.cn (X.J.); lihb@shzu.edu.cn (H.L.); \\ Tel.: +86-898-66961060 (X.J.); +86-993-2057912 (H.L.) \\ + These authors contributed equally to this work.
}

Received: 20 January 2019; Accepted: 26 February 2019; Published: 11 March 2019

\begin{abstract}
Myo-inositol-1-phosphate synthase (MIPS, EC 5.5.1.4) plays important roles in plant growth and development, stress responses, and cellular signal transduction. MIPS genes were found preferably expressed during fiber cell initiation and early fast elongation in upland cotton (Gossypium hirsutum), however, current understanding of the function and regulatory mechanism of MIPS genes to involve in cotton fiber cell growth is limited. Here, by genome-wide analysis, we identified four GhMIPS genes anchoring onto four chromosomes in G. hirsutum and analyzed their phylogenetic relationship, evolutionary dynamics, gene structure and motif distribution, which indicates that MIPS genes are highly conserved from prokaryotes to green plants, with further exon-intron structure analysis showing more diverse in Brassicales plants. Of the four GhMIPS members, based on the significant accumulated expression of GhMIPS1D at the early stage of fiber fast elongating development, thereby, the GhMIPS1D was selected to investigate the function of participating in plant development and cell growth, with ectopic expression in the loss-of-function Arabidopsis mips1 mutants. The results showed that GhMIPS1D is a functional gene to fully compensate the abnormal phenotypes of the deformed cotyledon, dwarfed plants, increased inflorescence branches, and reduced primary root lengths in Arabidopsis mips1 mutants. Furthermore, shortened root cells were recovered and normal root cells were significantly promoted by ectopic expression of GhMIPS1D in Arabidopsis mips1 mutant and wild-type plants respectively. These results serve as a foundation for understanding the MIPS family genes in cotton, and suggest that GhMIPS1D may function as a positive regulator for plant cell elongation.
\end{abstract}

Keywords: MIPS; exon-intron structure diversity; Gossypium hirsutum; loss-of-function mutant; root cell elongation

\section{Introduction}

Myo-inositol-1-phosphate synthase (MIPS; EC 5.5.1.4) is the rate-limiting enzyme to control myo-inositol (Ins) biosynthesis by converting D-glucose 6-phosphate (G6P) to inositol phosphate, followed by dephosphorylation reaction that is catalyzed by myo-inositol monophosphatase (IMP) [1]. The MIPS genes are widely spread in numerous organisms, from cyanobacteria to eubacteria and 
archaea, and ultimately to higher eukaryotes, such as higher plants and humans [2]. Numbers of MIPS have been identified in higher plants, including Phaseolus vulgaris [3], Oryza sativa [4], Citrus paradise [5], Arabidopsis thaliana [6], Zea mays [7], Brassica napus [8], Glycine max [9], and Sesamum indicum [10]. Protein sequence analysis of MIPS indicated that the eukaryotic MIPS family is homogenous, but different from the prokaryotic MIPS proteins [11]. This may be explained by the monophyletic origin of the eukaryotic MIPS genes. All eukaryotic MIPS sequences (around 510 amino acids) have shown regions of high conservation even at the nucleotide level [12], indicating that MIPS is very important for biological processes, such as embryogenesis and seed formation. There are four highly conserved amino acid sequences in MIPS proteins: GWGGNNG (domain 1), LWTANTERY (domain 2), NGSPQNTFVPGL (domain 3) and SYNHLGNNDG (domain 4) [1]. It is reported that these domains are involved in MIPS protein binding for catalyzing enzyme reaction and are essential for MIPS functions [2].

By catalyzing the biosynthesis of Ins and its derivates, including Ins polyphosphates (IPs), phospholipid phosphatidylinositol (PtdIns) and phosphoinositide phosphates (PtdInsPs), MIPS performs crucial diverse roles in biotic- and abiotic-stress responses, plant growth and organ development, and cell division [13]. The first MIPS was reported from Archaeoglobus fulgidus and might function under high temperatures [14]. In Arabidopsis, three genes encoding MIPS proteins (MIPS1-3) have been identified and appeared to have undergone functional divergence to some extent. Murphy et al. [15] found that loss-of-function Arabidopsis mips2 mutants showed enhanced susceptibility to diverse viral, bacterial, and fungal pathogens, whereas mips1 mutants showed no such effect. Furthermore, mips1 mutants exhibit spontaneous cell death and enhanced resistance to the oomycete pathogen [16]. The misp1 mutants were also reported to be sensitive to strong light stress [17]. The mips1 mips2 double mutant and mips1 mips2 mips3 triple mutant were embryo lethal, whereas the single mips mutants showed no obvious phenotype [18]. Severe reduction of MIPS activity inhibited plant growth significantly [19]. Lower $\mathrm{IP}_{6}$ generated by suppressing MIPS using RNA interference (RNAi)-mediated approach in potato led to the alteration of leaf morphology, induction of leaf senescence, and reduction of tuber yield [7].

Allotetraploid cotton Gossypium hirsutum is the most widely planted cotton species, which provide more than $90 \%$ of natural fiber for the textile industry. The yield of cotton fiber is greatly influenced by the fiber initiation and early stage of fiber elongation. Ins and its derivates also play important role in cell growth by involving in cell wall synthesis, membrane trafficking, and signal transduction. The oxidation product of Ins, D-glucuronic acid (GlcA), is used for cell wall pectic noncellulosic compounds and, in some organisms, for the synthesis of ascorbic acid (AsA) [20-24]. Pectin precursors were reported to be essential for cotton fiber elongation in tetraploid cotton G. hirsutum [25]. Our previous works presented that AsA and its oxidation metabolism catalyzed by ascorbate peroxidase (GhAPX) were important for cotton fiber development in G. hirsutum [26,27]. PtdIns and PtdInsP were significantly accumulated in fast elongating fibers and promoted fiber cell elongation in vitro [28]. GhMIPS genes were preferably expressed during fiber initiation and the early stage of fiber elongation development. Nonetheless, current understanding of cotton MIPS family genes and their functions involving in plant growth and development and fiber cell growth is limited. In this work, we identified four MIPS gene family members in G. hirsutum through a genome-wide investigation and characterized the detailed information of phylogenetic relationship, evolutionary dynamics, exon-intron structure, and conserved motif distribution. Furthermore, after consideration of the expression levels of the four GhMIPS genes during fiber development, we selected the GhMIPS1D to further investigate its functional roles in participating in plant development and cell growth by ectopic expression of GhMIPS1D in Arabidopsis mips1 mutants. The results indicated that GhMIPS1D could rescue the mutant abnormal phenotypes and ectopic expression of GhMIPS1D in mips1 mutant and wild-type (WT) Arabidopsis plants promoted the root cell elongation significantly. 


\section{Results}

2.1. Characterization, Phylogenetic Relationship and Motif Distribution Analyses of GhMIPS Gene Family Members in G. hirsutum

Cotton MIPS family genes were characterized by submitting the Arabidopsis MIPS protein sequences against G. hirsutum genome (allotetroploid, AADD) database derived from Phytozome (v12.1.6), followed by conserved domain recognition using InterProScan. Four GhMIPS members were determined: GhMIPS1A, GhMIPS2A, GhMIPS1D and GhMIPS2D (the sub-genomes were indicated by A and D). The GhMIPS1A located in sub-genome A chromosome 2 while GhMIPS1D located in sub-genome D chromosome 3, indicating homologous recombination events might occur during the individual evolution of A and D sub-genomes (Supplementary Figure S1). Due to the highly conserved gene sequences and structures, all GhMIPS homologues were considered as duplicates and the duplication relationships were indicated by red lines in Supplementary Figure S1. The Ka/Ks analysis showed that all GhMIPS members are under purifying selection (Supplementary Table S1). Expression patterns of GhMIPS family genes showed that GhMIPS1A and GhMIPS1D were the most highly expressed members in cotton fibers, with the highest expression level at 0 day post anthesis (DPA) (no fiber growth) and 3 DPA (fiber initiation growth) (Supplementary Figure S2), implying that GhMIPS1D may perform potential important role in fiber growth and development.

Phylogenetic tree of 70 MIPS homologues of 36 different species from prokaryotes to higher plants was constructed to investigate the evolutionary characters of the MIPS gene family. In general, seven prokaryotes, seven animal, six fungus and fifty plant MIPS proteins were included for the phylogenic analysis. Detailed information of MIPS used in this study is provided (Supplementary Table S2), containing organism names, accession numbers and sequences. The MIPS gene family is a very small one, usually including one or two members in most plant species, except for Z. mays (diploid, monocotyledons); Brassica rapa, Brassica oleracea and Glysine max (diploid, dicotyledons); G. hirsutum and Gossypium barbadense (tetraploid, dicotyledons); which have four MIPS members each. In addition to B. rapa and B. oleracea, the model plant $A$. thaliana has three MIPS members, indicating that this protein family has expanded in most Brassicales plants (Figure 1). 


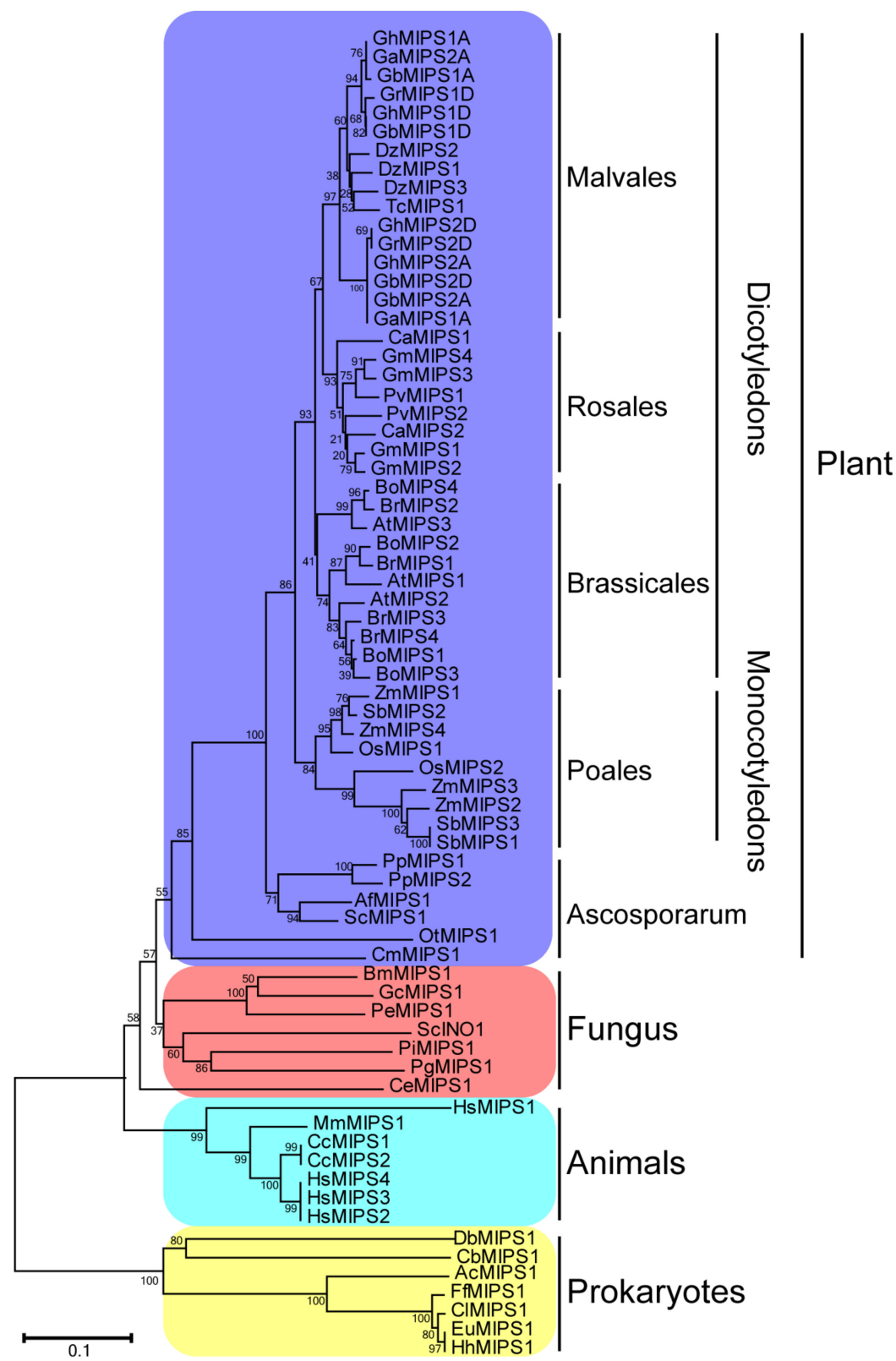

Figure 1. Phylogenetic tree of 70 MIPS homologues of 36 different species from prokaryotes to higher plants. See Supplementary Table S2 for detailed information of organism and protein sequences. The phylogenetic tree was constructed by MEGA5.0 using neighbor-joining method with bootstrap tests 1000 .

GhMIPS1D and other nine typical plant MIPS proteins were used to perform multiple sequence alignment and conserved domain analysis, showing that MIPS proteins are quite conserved in plants with amino acid identity over $80 \%$. Similar with the other nine plant MIPS proteins, cotton GhMIPS1D possessed two NAD(P)-binding domains distributing at the $\mathrm{N}$ and $\mathrm{C}$ terminus respectively, and one myo-inositol-1-phosphate synthase domain locating at the middle region, as well as four conserved domains that are responsible for MIPS protein binding and are essential for MIPS function exertion (Figure 2). Note that in higher plants, all MIPS proteins have around 510 amino acids, while CmMIPS1 of simple single cell algae (Cyanidioschyzon merolae) has 530 amino acids (Figure 2). Motif distribution analysis recognized ten conserved motifs that are organized similarly in all plant MIPS proteins (Figure 3A and see Supplementary Figure S3 for detailed sequences for motifs). These data suggest that MIPS is a highly conserved protein family in different plants, from ascosporarum to higher plants. 

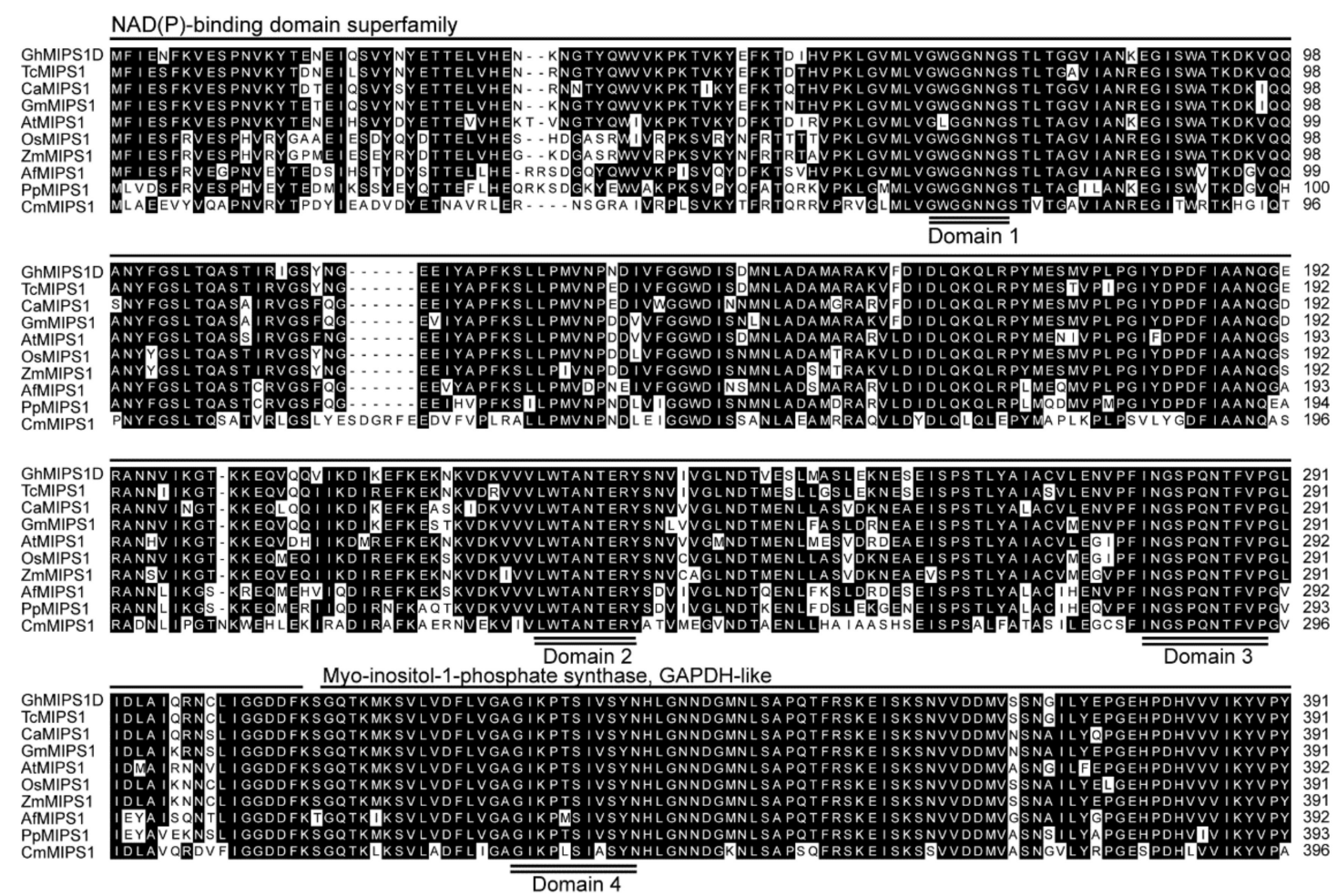

$\mathrm{NAD}(\mathrm{P})$-binding domain superfamily
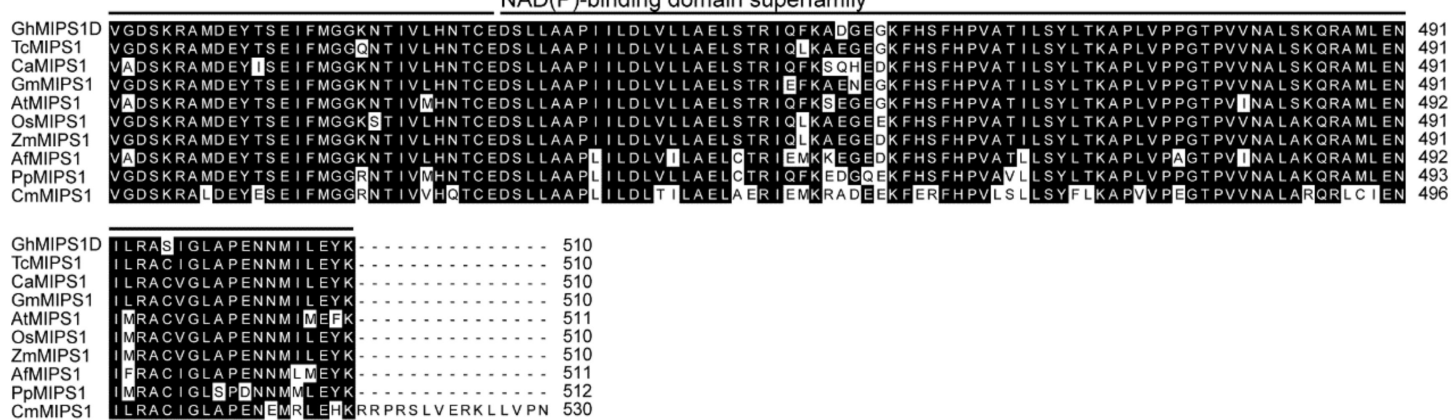

Figure 2. Multiple sequence alignment of ten representative MIPS proteins. See Supplementary Table S2 for organism and sequence information. Conserved domains are indicated with double underline (Domain 1-4). 
A

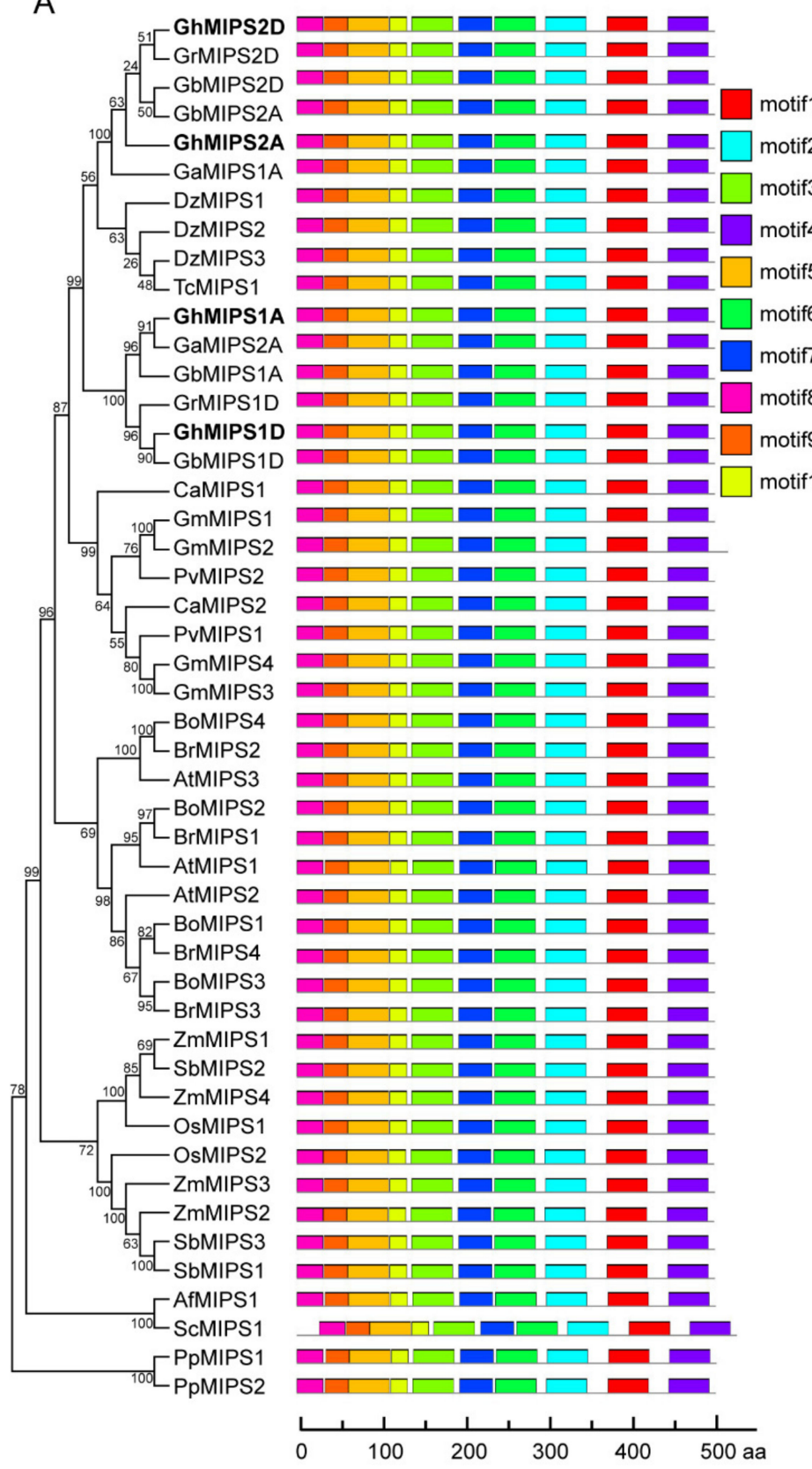

B

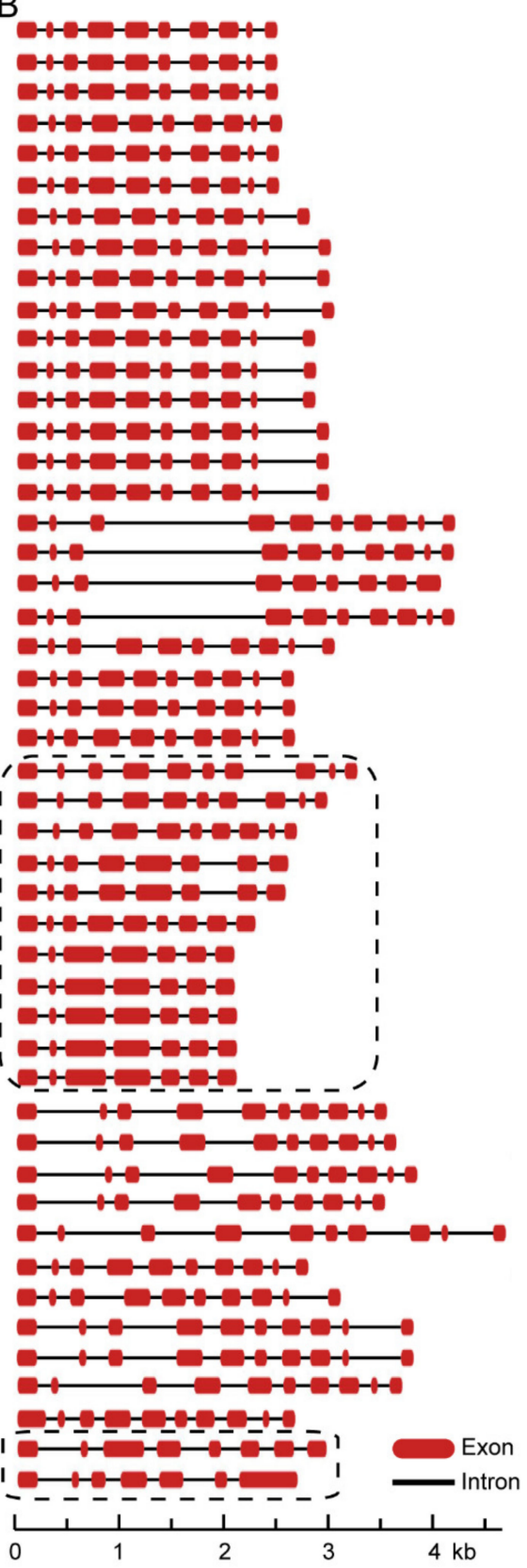

Figure 3. Conserved motif and exon-intron dynamic analyses of 48 plant MIPS genes. (A) Conserved motifs of protein sequences of 48 plant MIPS proteins. Ten conserved motifs were recognized and represented in different colors. All MIPS proteins are arranged according to their phylogenetic relationships. (B) The exon-intron structures of 48 plant MIPS genes. Two frames represent Bryophytes plants (Physcomitrella patens, seven and eight exons) and Brassicales plants (from seven to ten exons), which possess usual exon-intron structures. The detailed information for organism names, protein sequences and accession numbers are available in Supplementary Table S2.

\subsection{Exon-Intron Structure Evolution Analysis of MIPS Gene Family}

To further study the evolutionary relationship of MIPS family members, exon-intron structure dynamic analysis of four cotton GhMIPS members and other 44 plant MIPS genes was performed. Only one exon was found in single-cell algae C. merolae and Ostreococcus tauri. However, Physcomitrella patens PpMIPS1 and PpMIPS2 possess eight and seven exons respectively, indicating that gene family expansion and gene structure variation occurred after the terrestrial plant has been evolved (Figure 3B). 
For higher plants, all MIPS proteins have ten exons except B. rapa, B. oleracea and A. thaliana, suggesting that Brassicales plants have undergone some special evolutionary events (Figure 3B, framed).

According to the phylogenetic tree and the exon-intron structures, an evolution model of the exon-intron structures for plant MIPS gene family was illustrated. The single-exon structure had been split into seven and eight exons shortly after the appearance of land plants. Then the last exon of the seven-exon structure homologues split into four new exons (event I, Figure 4A), while the 3rd and the last exons of the eight-exon structure members split into two new exons each (event II and event III, Figure 4A). Red arrows in Figure 4A indicate the exon split sites and black arrows indicate the amino acid sequences corresponding to the final exon-intron structures. Our data show that the formation of the ten-exon structure of MIPS genes is no later than arise of ferns (Azolla filiculoides and Salvinia cucullata).

A

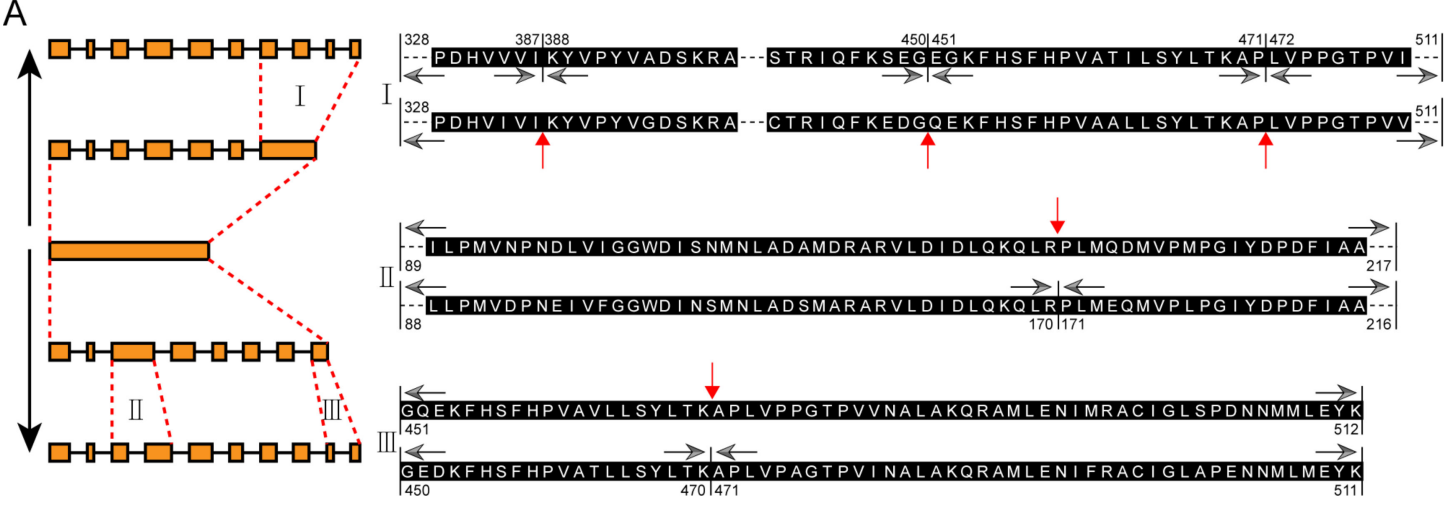

B

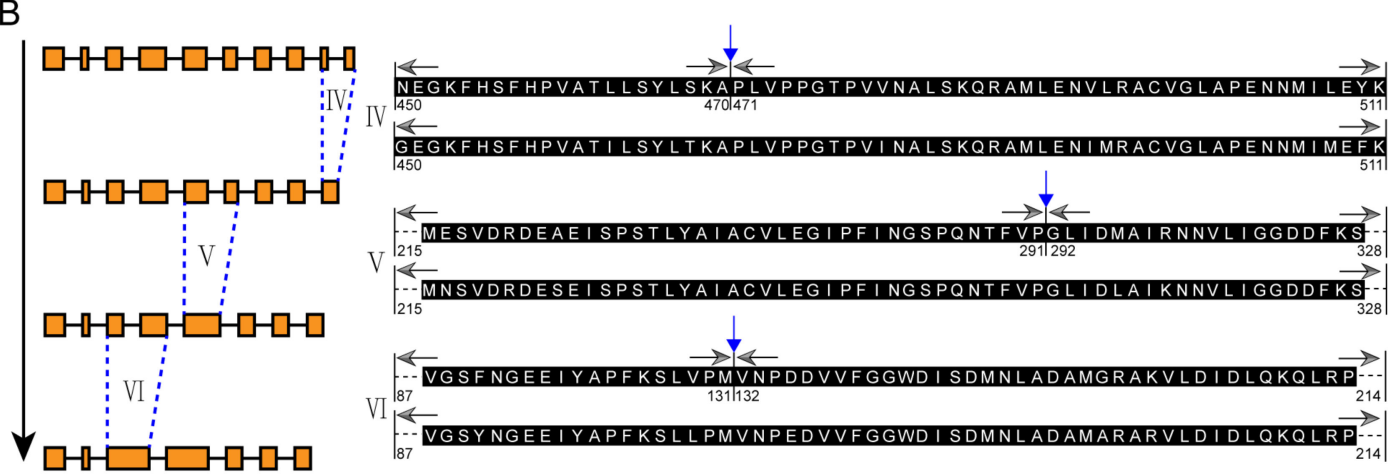

Figure 4. Illustration of the evolution model of MIPS exon-intron structures. (A) The evolution model of exon split events. MIPS possesses one entire exon in single cell algae. One evolution event has been recognized in Bryophytes plants, leading to a seven-exon PpMIPS2 (up-arrow) and an eight-exon PpMIPS1 (down-arrow). Then some of the exons have been split into more exons, forming the predominant exon-intron structure in higher plants (events I, II and III). The exon split sites are indicated by red arrows and amino acid numbers. (B) The evolution model of exon merge events in Brassicales plants. Three exon merge events have been identified, indicating there were three independent evolutionary events during the evolution of Brassicales plants. The exon-merge sites are indicated by blue arrows.

Notably, three exons merge events were recognized in Brassicales plants, leading to the formation of nine-exon (AtMIPS1), eight-exon (BoMIPS2 and BrMIPS1) and seven-exon (AtMIPS2, BoMIPS1, BoMIPS3, BrMIPS3 and BrMIPS4) MIPS family members, respectively (Figure 4B, and Figure 3B). Interestingly, the three exons merge events seem to occur in order (from event IV to event VI), indicating the Brassicales plants MIPS might have undergone three special evolutionary events. 


\subsection{Functional Complementary Analysis of GhMIPS1D in the Loss-of-Function Arabidopsis mips1 Mutant}

In the light of Ins and its derivates important functions in plant development and cell growth [13], as well as the accumulated expression of GhMIPS1D during cotton fiber development (Supplementary Figure S2), thus, the overexpression vector 35S::GhMIPS1D-GFP generated by cloning the GhMIPS1D into the modified $p C A M B I A 2300-G F P$ vector, was transformed into the loss-of-function mips1 mutant and WT Arabidopsis plants, to investigate the GhMIPS1D in vivo functions. The 35S::GhMIPS1D-GFP vector was introduced into onion epidermal cells to determine the subcellular localization of GhMIPS1D. The results showed that GhMIPS1D located in the nucleus, plasma membrane and endomembranes (Supplementary Figure S4), suggesting its likely functions in membrane trafficking and signal transduction, and regulation of gene expression.

The loss-of-function Arabidopsis mips1 mutants were selected to perform genetic functional analysis of GhMIPS1D. The expression levels of GhMIPS1D in the transgenic Arabidopsis lines were examined (Supplementary Figure S5). As is reported, the T-DNA insertion Arabidopsis mutant lines exhibit abnormal cotyledon development (Figure 5B), shorter plants (Figure 5F) and increased inflorescence branch numbers (Figure 5G), and sensitivity to strong light stress (Supplementary Figure S6). The transgenic mips1/GhMIPS1D lines, obtained by transforming 35S::GhMIPS1D-GFP vector into Arabidopsis mips1 mutants, indicated stable expression levels of GhMIPS1D and rescued the aberrant phenotypes of Arabidopsis mips1 mutants (Figure 5C-G). In addition, the Arabidopsis mutant lines expressing GhMIPS1D also showed increased tolerance to light stress under $220 \mu \mathrm{mol} \cdot \mathrm{m}^{-2} \mathrm{~s}^{-1}$ (Supplementary Figure S6). These data suggest that GhMIPS1D is the predominantly expressed MIPS gene in G. hirsutum and is an active gene effectively to rescue the abnormal defects of growth and development in Arabidopsis mips1 mutants. 

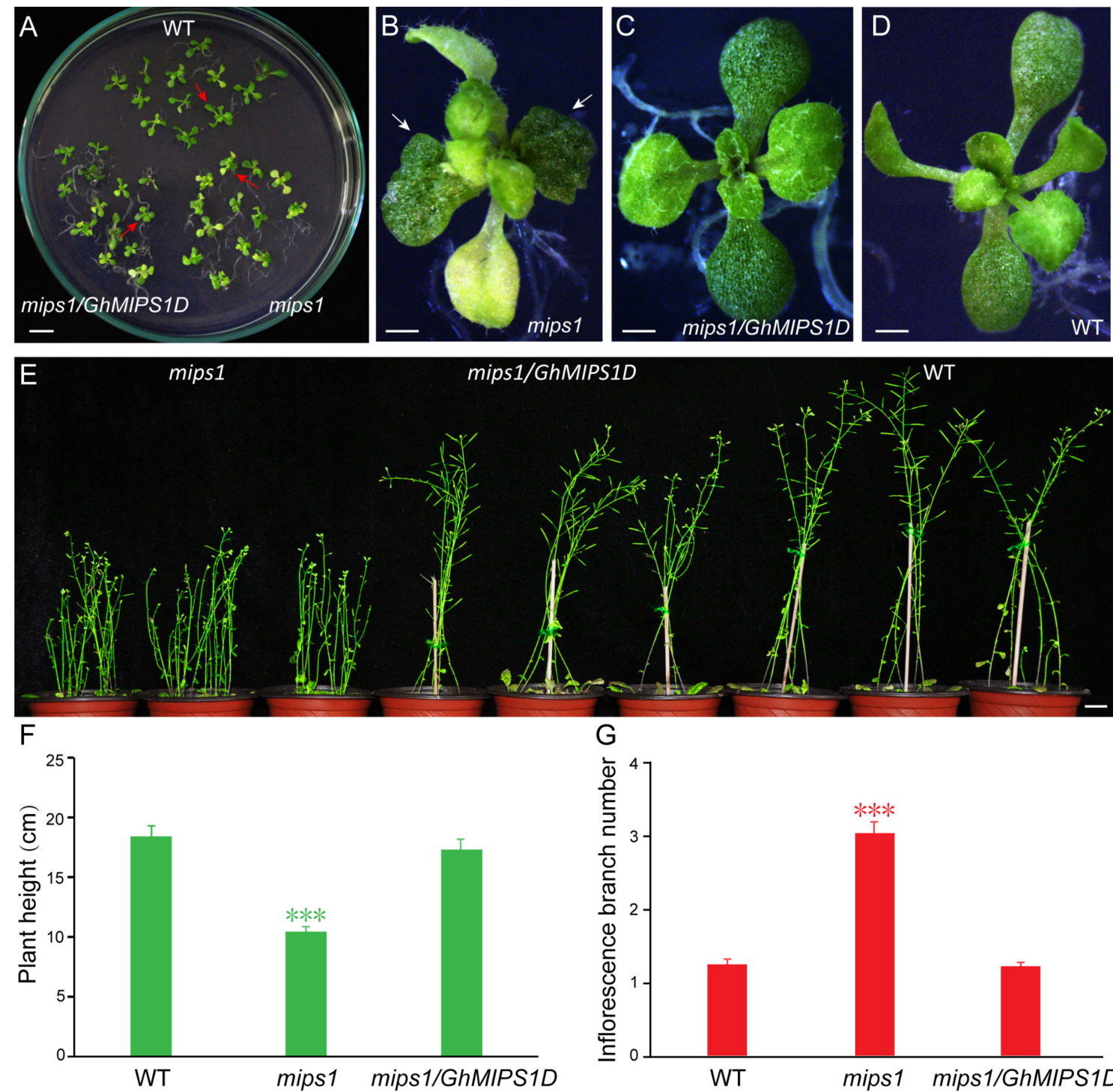

S1/GhMIPS1D
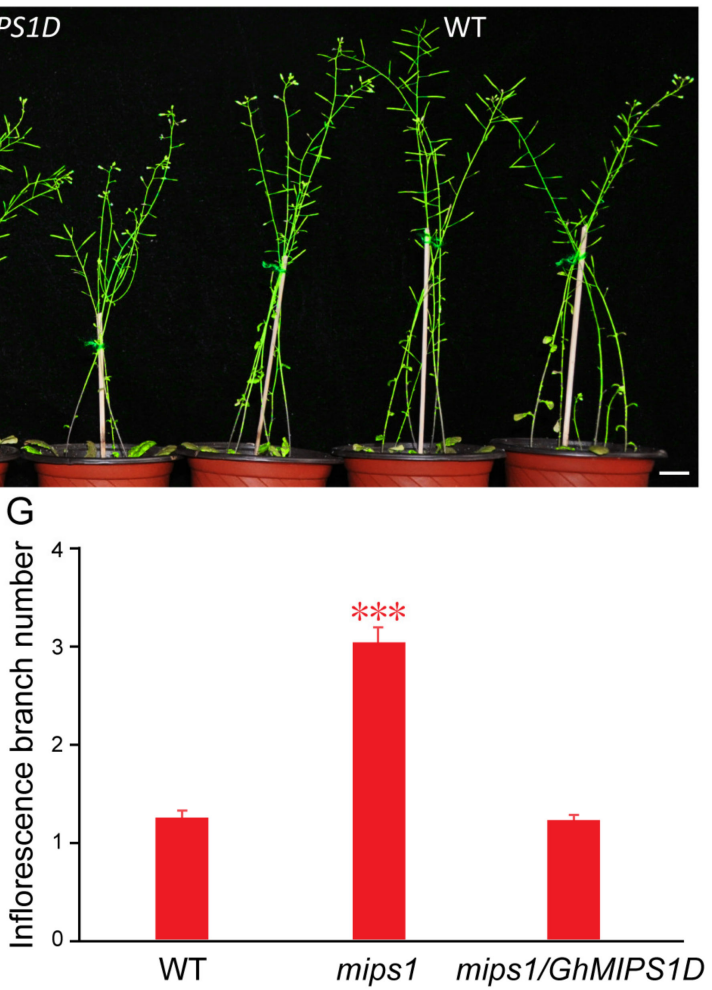

Figure 5. Ectopic expression of GhMIPS1D in Arabidopsis rescues the abnormal defects of mips1 mutants. (A) The mips1 mutant (mips1), transgenic (mips1/GhMIPS1D) and wild-type (WT) Arabidopsis plants grew on $0.5 \times$ Murashige and Skoog (MS) medium for two weeks. Bar $=1 \mathrm{~cm}$. (B-D), the enlarged image of indicated plants (red arrows in (A)). White arrows indicate the abnormal cotyledon in mips1 mutants. Bars $=1 \mathrm{~mm}$. (E) Phenotypes of Arabidopsis plants grew for ten weeks. Significant shorter plants and more inflorescence branches are observed in mips1 mutants, but not in mips1/GhMIPS1D transgenic lines and WT plants. Bar $=1 \mathrm{~cm}$. The plant height $(\mathbf{F})$ and the number of inflorescence branches $(\mathbf{G})$ are measured $(n=30) .{ }^{* * *}, p<0.001$ compared to WT.

\subsection{Ectopic Expression of GhMIPS1D Promotes Root Cell Elongation in Arabidopsis}

Considering that the Ins derivates play essential roles in root development and root hair formation [29,30], and GhMIPS1D gene highly expressed during the stages of fiber initiation and early elongation (Supplementary Figure S2), which indicates its potential important function for plant cell elongation. Thus, we further measured the length of primary roots and root cells in Arabidopsis lines ectopically expressing GhMIPS1D. The transgenic mips1/GhMIPS1D plant lines exhibited normal root length similar to that in WT, suggesting that GhMIPS1D is a functional gene to compensate the root elongation development defect of Arabidopsis mips1 mutants (Figure 6A,B). In addition, the transgenic plants ectopically expressing GhMIPS1D in WT Arabidopsis demonstrated significantly increased primary root length (Figure 6A,B). Further confocal microscopy detection displayed that the primary 
root cell lengths are increased from $124.02 \pm 10.01 \mu \mathrm{m}$ (WT) to $152.14 \pm 16.58 \mu \mathrm{m}$ (35S::GhMIPS1D), indicating that GhMIPS1D is essential for plant cell elongation (Figure 6C,D). Totally, the genetically functional complementary analyses suggest that GhMIPS1D is a fully functional MIPS and plays as a key positive regulator to involve in cell elongation.

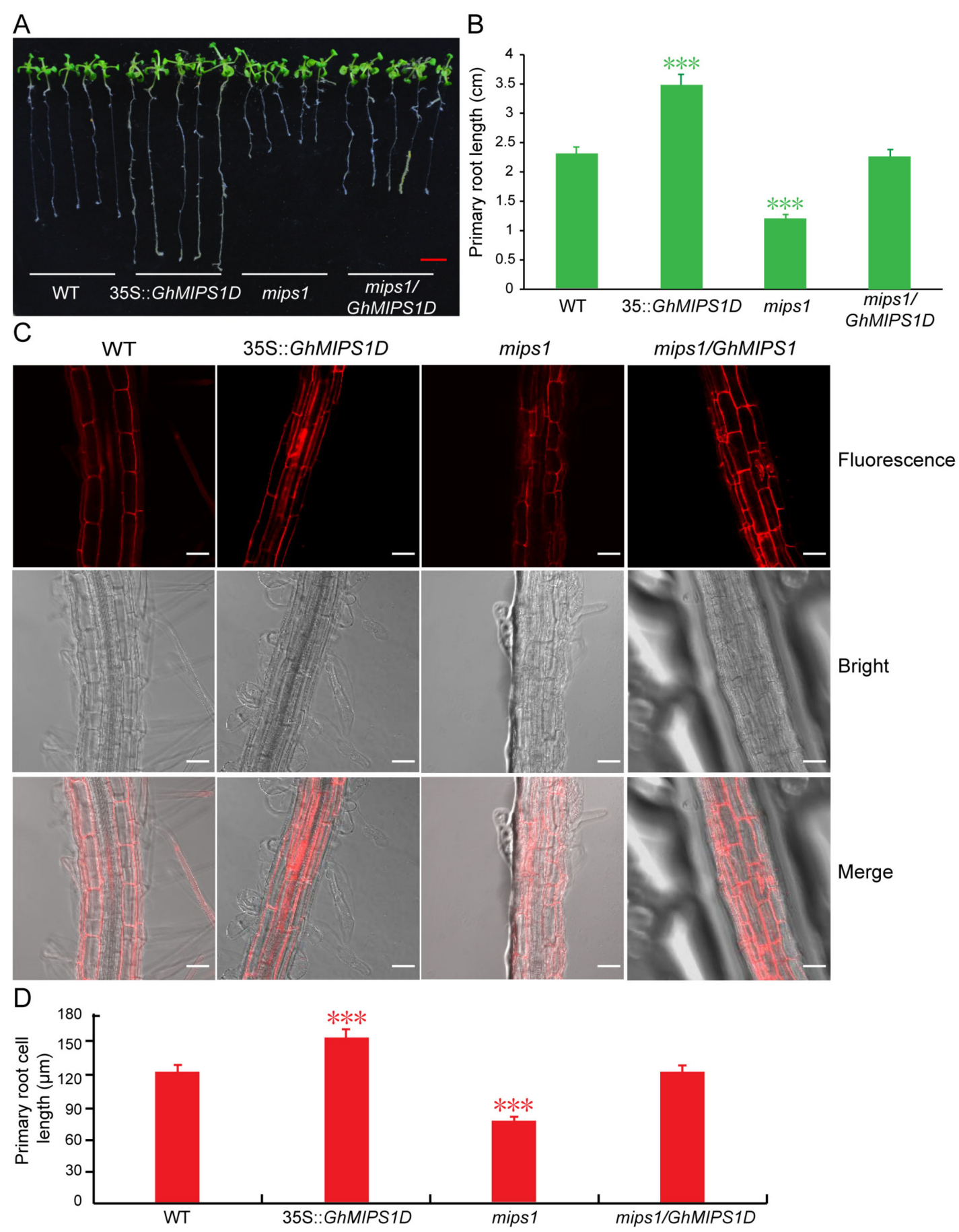

Figure 6. Ectopic expression of GhMIPS1D promotes Arabidopsis root cell elongation. (A) The primary root phenotypes of wild-type (WT), transgenic lines ectopically expressing GhMIPS1D (35S::GhMIPS1D), mips1 mutants (mips1) and transgenic lines expressing GhMIPS1D in mips1 mutants (mips1/35S::GhMIPS1D). Red bar $=0.5 \mathrm{~cm}$. (B) The primary root lengths are measured $(n=10)$. (C) Fluorescence images of Arabidopsis root cells under confocal microscopy. Bars $=50 \mu \mathrm{m}$. (D) The root cell lengths are measured $(n=50)$. The results show that ectopic expression of GhMIPS1D significantly induces the cell length in Arabidopsis roots. ${ }^{* * *}, p<0.001$ compared to WT. 


\section{Discussion}

Lots of MIPS genes have been identified in numerous living organisms, such as bacteria, fungi, animals, and higher plants, etc. [31]. Generally, there are one or two MIPS members in most plant species, with the exception of three members in A. thaliana, and of four members in Z. mays, B. rapa, B. oleracea and G. max, respectively. In this study, by genome-wide analysis, we identified four G. hirsutum MIPS family genes that are distributed across different chromosomes (Supplementary Figure S1). Phylogenetic tree of 70 MIPS from 36 species was constructed and used for evolutionary analysis, benefiting from the dramatically increased genome database. The protein sequences of eukaryotic MIPS exhibit high conservation, supporting the explanation that eukaryotic MIPS genes have a monophyletic origin (Figure 2). However, the single-cell algae C. merolae and Ostreococcus tauri possess MIPS proteins of 533 and 535 amino acids, while all higher plants have MIPS proteins of around 510 amino acids (from 509 to 511), displaying that sequence lost events had occurred after plant landing. Only two higher plant MIPS proteins possess amino acids much longer than 510 amino acids: S. cucullate MIPS1 (537 amino acids) and G. max MIPS2 (526 amino acids) (Supplementary Table S2), indicating special evolutionary events in these two species. Many studies reported the conservation of MIPS protein sequences, but few noticed the exon-intron structure evolution patterns of MIPS genes [32,33]. The exon-intron structure analysis presented here suggested that the MIPS gene structures were highly conserved in higher plants except for Brassicales, suggesting that MIPS family genes underwent more complicated evolutionary events during the Brassicales plants evolution (Figures 2 and 3). This may explain the fact that Arabidopsis MIPS family members show function diversity $[17,18,31,34]$.

MIPS is the key biosynthetic enzyme for the formation of Ins and its derivates, including PtdIns and PtdInsP, which have been proven as a crucial regulator to control plant growth and organ development. Of the four GhMIPS members, GhMIPS1D, showing the predominantly expression during the early stages of fiber development (Supplementary Figure S2), was selected to further investigate cotton MIPS functions of participating in plant growth and development. After ectopic expression of GhMIPS1D in Arabidopsis loss-of-function mips1 mutants, the aberrant phenotypes of cotyledon abnormality, shorter plants, increased inflorescence branches and sensitivity to light in the mips 1 mutants were rescued significantly (Figure 5 and Supplementary Figure S6), implying the cotton MIPS important roles in the involvement of plant growth and development as the pivotal enzyme to produce Ins and its derivates that are important signal molecules of the cell. Knockout of the soybean GMMIPS1 affected the early development of the embryo and resulted in the termination of seed mature that might be caused by the reduction of $\mathrm{IP}_{6}$ [35]. Arabidopsis MIPS1 is essential for seed development, and the loss of MIPS1 leads to the reduction of AsA and PtdIns and thus produces the irregular phenotypes of smaller plants, curly leaves and generation of lesions [18]. Ins produced by MIPS is crucial for Arabidopsis embryogenesis through regulating the synthesis of PtdIns and phosphatidylinositides and thereby involving in endomembrane structure trafficking and PIN1-mediated auxin singaling pathway [17]. The functions of the other GhMIPS genes should also be verified in the future for a better understanding of the diverse roles of Ins pathway in cotton. Besides, MIPS has also been reported to function in salt and drought tolerance, indicating that the MIPS gene family play various roles in plant development $[36,37]$. However, there still are unsolved problems should be addressed in the future, such as the mechanism leading to increased inflorescence branch numbers in Arabidopsis misp1 mutants, as well as the possible phenotypes of loss-of-function cotton mips1 and/or mips2 mutants.

Due to the highly conserved protein sequences of GhMIPS1D and AtMIPS1, it is not surprising that GhMIPS1D could rescue the Arabidopsis mips1 mutant phenotypes. As is well known, MIPS proteins from diverse species share highly conserved core catalytic domains, which endow the activity to catalyze rate-limiting redox reaction to generate the Ins formation from G6P [31]. The G. hirsutum MIPS possessed several typical domains that serve as binding and catalysis of MIPS proteins (Figure 2, Supplementary Figure S3). Since the first MIPS was reported from Archaeoglobus fulgidus, many MIPS 
proteins have been investigated from different organisms. The MIPS in higher organisms usually showed cytosolic or organellar location. In this study, subcellular distribution analysis indicated the location of GhMIPS1D in the nucleus, plasma membrane and endomembranes (Supplementary Figure S4), suggesting that cotton MIPS may perform a diverse role in different compartments to participate in plant growth and development. By suppressing the spreading of heterochromatin, MIPS can bind to its promoter to induce its own expression, providing the illustration of the regulatory mechanism at the transcriptional level [38].

MIPS catalyzed Ins biosynthesis provides the important supply for synthetizing its derivates containing PtdIns, PtdInsPs and InsPs that are vital signal molecules to affect cell growth by participating in cellular signal transduction [39]. In this study, GhMIPS1D indicated preferential expression during fiber initial growth stage (Supplementary Figure S2), and ectopic expression of GhMIPS1D in Arabidopsis rescued the shorter primary root lengths in mips1 mutants and promoted the root cell elongation in WT, respectively (Figure 6). Severe reduction of $\mathrm{IP}_{6}$ content in Arabidopsis ipk1-1 mutants significantly inhibits the root hair elongation [40]. The decrease of $\mathrm{PtdInsP}_{2}$ content in Arabidopsis results in suppression of pollen tube elongation by affecting apical pectin deposition and membrane trafficking [41]. Although many Ins derivates have been reported to be involved in cell growth, whereas, this is the first time to prove that MIPS family members could promote the root cell elongation in Arabidopsis (Figure 6). This phenomenon is reasonable for that previous work showed that pectin and AsA play important roles in cotton fiber elongation $[25,26]$. MIPS has been reported to increase the synthesis of pectin precursor of UDP-D-glucuronic acid (UDP-GlcA) and AsA [42]. UDP-GlcA, PtdIns and PtdInsP that are important Ins derivates have been studied to involve in fiber elongation growth as important components of the cell wall and cell membranes [25,28].

De novo synthesis of Ins is required for the correct transport and localization of auxin during embryo pattern formation in Arabidopsis [17,18]. The functions of MIPS during cotton fiber development has not been well studied before. Many studies have reported that auxin signaling is essential for the initiation and early elongation of cotton fiber [43,44]. In the present study, GhMIPS1D was highly expressed at the early stage of cotton fiber development (Supplementary Figure S2), providing the potential possible direct or indirect link between MIPS catalyzed synthesis of Ins and its derivates and auxin signal transduction. Arabidopsis mips1 mutants exhibit a significant reduction of PtdIns content and decrease of auxin polar transport rate that regulates the cellular auxin distribution [45]. Further investigation on MIPS and phytohormone crosstalk will provide a better understanding of functions of MIPS during cotton fiber development in the future.

In conclusion, we identified four MIPS family gene members in G. hirsutum, the phylogenic relationship, multiple sequence alignment, and motif distribution analyses showed that the MIPS gene family is highly conserved. Further genetically functional complementary analysis showed that GhMIPS1D is the predominantly expressed MIPS gene in cotton fibers, and ectopic expression of GhMIPS1D in Arabidopsis mips1 mutant rescues the phenotypes of abnormal cotyledon development, increased inflorescence branch numbers and light stress sensitivity. Moreover, ectopic expression of GhMIPS1D in WT Arabidopsis promotes root cell elongation significantly. Our results provide a genome-wide analysis of the MIPS gene family in G. hirsutum and suggest that the GhMIPS1D is a positive regulator involving in plant cell elongation.

\section{Materials and Methods}

\subsection{Sequence Acquirement and Chromosomal Distribution of GhMIPS Genes}

The prokaryotic MIPS protein sequences were retrieved from NCBI (database downloaded on 31 December 2018) by online BLASTP. The eukaryotic MIPS protein sequences were determined using local BLASTP program by submitting Arabidopsis MIPS to the genome database for each organism. The genome data were downloaded from the online database Phytozome (v12.1.6). The candidate MIPS proteins were confirmed by screening the conserved domains using InterProScan. The genomic 
location of GhMIPS genes was performed using local BLASTN against the G. hirsutum genome database. Mapinspect was used to draw the location of MIPS genes in different chromosomes.

\subsection{Phylogenic and Evolutionary Analyses}

Multiple sequence alignment was performed using ClustalW and the phylogenetic tree was constructed by MEGA5.0 [46] using the neighbor-joining method with bootstrap tests 1000 . The evolutionary analyses were performed as previously described [47,48]. Briefly, the exon-intron distribution analysis was performed using the gene structure display server (GSDS, http:/ / gsds. cbi.pku.edu.cn/index.php). Conserved motifs were recognized by MEME online software (http: //meme-suite.org/tools/meme). The Ka/Ks was calculated by Dnasp (v6) software [49].

\subsection{Plant Materials}

The Arabidopsis mips1 mutant (SALK_023626) was obtained from Lijia Qu, State Key Laboratory of Protein and Plant Gene Research, College of Life Sciences, Peking University. All Arabidopsis plants were grown on $0.5 \times$ MS medium as previously described [17].

\subsection{Construction of Vectors, Subcellular Localization Analysis and Ectopic Expression of GhMIPS1D in Arabidopsis}

The full-length of the coding sequences of GhMIPS1D gene was amplified using primers in Supplementary Table S3, and then cloned into the modified pCAMBIA2300-GFP vector using KpnI and $\mathrm{XbaI}$ to generate 35S::GhMIPS1D-GFP, which was used for further ectopic expression and subcellular localization analyses. The successfully constructed vectors were then transformed into Agrobacterium tumefaciens stain GV3101.

The subcellular localization analysis was performed using onion epidermal cells and A. tumefaciens containing 35S::GhMIPS1D-GFP vector. Briefly, the inside epidermal layer of the onion was separated. After soaking in $75 \%$ ethanol for $10 \mathrm{~min}$ and washing 3-4 times with sterile water, the inside epidermis was cut and co-cultivated with A. tumefaciens containing 35S::GhMIPS1D-GFP vector on $1 / 2 \mathrm{MS}$ at $28{ }^{\circ} \mathrm{C}$ under darkness. After a sub-culturing for $24 \mathrm{~h}$, a confocal laser-scanning microscope (Zeiss LSM510, Oberkochen, Germany) was used to detect the GFP signals with an activation wavelength of $488 \mathrm{~nm}$.

Wild-type (WT) and mips1 mutant Arabidopsis plants were transformed using A. tumefaciens stain GV3101 containing 35S::GhMIPS1D-GFP vector. The functional analysis of transgenic Arabidopsis was performed as reported before [50].

\subsection{Statistical Analysis}

All statistics were performed by One-way ANOVA followed by Bonferroni test using SigmaStat software (Version 4.0) (Starcom Information Technology Ltd, Bangalore, India). ${ }^{* * *}$ represents significant difference at $p<0.001$ level.

Supplementary Materials: Supplementary materials can be found at http://www.mdpi.com/1422-0067/20/5/ 1224/s1.

Author Contributions: Conceptualization, X.J. and H.L.; Methodology, X.J.; Software, R.M., W.S. and F.W.; Validation, R.M., W.S. and F.W.; Investigation, A.C., S.X. and X.C.; Resources, H.L.; Data Curation, X.J.; Writing-Original Draft Preparation, W.S. and X.J.; Writing—Review and Editing, F.W. and H.L.; Visualization, W.S., F.W., R.M. and A.C.; Supervision, X.J. and H.L.; Project Administration, R.M., W.S. and X.J.; Funding Acquisition, H.L.

Funding: This work was supported by grants of the National Natural Science Foundation of China (Grant number 31660408, 31260339) and Scientific and Technological Achievement Transformation Project of Bingtuan (Grant number 2016AC017).

Acknowledgments: The authors thank Lijia Qu from State Key Laboratory of Protein and Plant Gene Research, College of Life Sciences, Peking University for kindly providing the Arabidopsis mips1 mutant. We also 
thank Xianzhong Huang from the College of Life Sciences, Shihezi University for providing the modified vector PCAMBIA2300-GFP.

Conflicts of Interest: The authors declare no conflicts of interest.

\section{Abbreviations}

$\begin{array}{ll}\text { APX } & \text { Ascorbate peroxidase } \\ \text { AsA } & \text { Ascorbic acid } \\ \text { BLAST } & \text { Basic local alignment search tool } \\ \text { DPA } & \text { Day post anthesis } \\ \text { G6P } & \text { D-glucose 6-phosphate } \\ \text { GlcA } & \text { D-glucuronic acid } \\ \text { IMP } & \text { Myo-inositol monophosphatase } \\ \text { Ins } & \text { Myo-inositol } \\ \text { IP } & \text { Myo-inositol polyphosphate } \\ \text { MEGA } & \text { Molecular evolutionary genetics analysis } \\ \text { MIPS } & \text { Myo-inositol-1-phosphate synthase } \\ \text { MS } & \text { Murashige and Skoog } \\ \text { PtdIns } & \text { phospholipid phosphatidylinositol } \\ \text { PtdInsP } & \text { phosphoinositide phosphate } \\ \text { RNAi } & \text { RNA interference } \\ \text { UDP-GlcA } & \text { UDP-D-glucuronic acid } \\ \text { WT } & \text { Wild-type }\end{array}$

\section{References}

1. Majumder, A.L.; Chatterjee, A.; Dastidar, K.G.; Majee, M. Diversification and evolution of L-myo-inositol 1-phosphate synthase. FEBS Lett. 2003, 553, 3-10. [CrossRef]

2. Majumder, A.L.; Johnson, M.D.; Henry, S.A. 1-L-myo-inositol-1-phosphate synthase. Biochim. Biophys. Acta 1997, 1348, 245-256. [CrossRef]

3. Johnson, M.D.; Wang, X. Differentially expressed forms of 1-L-myo-inositolphosphate synthase (EC5.5.1.4) in Phaseolus vulgaris. J. Biol. Chem. 1996, 271, 17215-17218. [CrossRef] [PubMed]

4. Hait, N.C.; Chaudhuri, A.R.; Das, A.; Bhattacharyya, S.; Majumder, A.L. Processing and activation of chloroplast L-myo-inositol 1-phosphate synthase from Oryza sativa requires signals from both light and salt. Plant Sci. 2002, 162, 559-568. [CrossRef]

5. Abu-abied, M.; Holland, D. The gene c-ino1 from Citrus paradise is highly homologous to tur1 and ino1 from the yeast and Spirodela encoding for myo-inositol phosphate synthase. Plant Physiol. 1994, 106, 1689. [CrossRef] [PubMed]

6. Johnson, M.D.; Sussex, I.M. 1-L-myo-inositol 1-phosphate synthase from Arabidopsis thaliana. Plant Physiol. 1995, 107, 613-619. [CrossRef] [PubMed]

7. Keller, R.; Brearley, C.A.; Trethewey, R.N.; Muller-Rober, B. Reduced inositol content and altered morphology in transgenic potato plants inhibited for 1-D-myo-inositol 3-phosphate synthase. Plant J. 1998, 16, 403-410. [CrossRef]

8. Larson, S.R.; Raboy, V. Linkage mapping of maize and barley myo-inositol 1-phosphate synthase DNA sequences: Correspondence with low phytic acid mutation. Theor. Appl. Genet. 1999, 99, 27-36. [CrossRef]

9. Iqbal, M.; Afzal, A.; Yaegashi, S.; Ruben, E.; Triwitayakorn, K.; Njiti, V.; Ahsan, R.; Wood, A.; Lightfoot, D. A pyramid of loci for partial resistance to Fusarium solani $f$. sp. glycines maintains myo-inositol-1-phosphate synthase expression in soybean roots. Theor. Appl. Genet. 2002, 105, 1115-1123. [CrossRef] [PubMed]

10. Chun, J.A.; Jin, U.H.; Lee, J.W.; Yi, Y.B.; Hyung, N.I.; Kang, M.H.; Pyee, J.H.; Suh, M.C.; Kang, C.W.; Seo, H.Y.; et al. Isolation and characterization of a myo-inositol 1-phosphate synthase cDNA from developing sesame (Sesamum indicum L.) seeds: Functional and differential expression, and salt-induced transcription during germination. Planta 2003, 216, 874-880. [PubMed]

11. Bachhawat, N.; Mande, S.C. Complex evolution of the inositol-1-phosphate synthase gene among archaea and eubacteria. Trends Genet. 2000, 16, 111-113. [CrossRef] 
12. Hegeman, C.E.; Good, L.L.; Grabau, E.A. Expression of D-myo-inositol-3-phosphate synthase in soybean implication for phytic acid biosynthesis. Plant Physiol. 2001, 125, 1941-1948. [CrossRef] [PubMed]

13. Gillaspy, G.E. The cellular language of myo-inositol signaling. New Phytol. 2011, 192, 823-839. [CrossRef] [PubMed]

14. Chen, L.J.; Zhou, C.; Yang, H.Y.; Roberts, M.F. Inositol 1-phosphate synthase from Archaeoglobus fulgidus is a class II aldolase. Biochemistry 2000, 39, 12415-12423. [CrossRef] [PubMed]

15. Murphy, A.M.; Otto, B.; Brearley, C.A.; Carr, J.P.; Hanke, D.E. A role for inositol hexakisphosphate in the maintenance of basal resistance to plant pathogens. Plant J. 2008, 56, 638-652. [CrossRef] [PubMed]

16. Meng, P.H.; Raynaud, C.; Tcherkez, G.; Blanchet, S.; Massoud, K.; Domenichini, S.; Herry, Y.; Soubigou-Taconnat, L.; Lelarge-Trouverie, C.; Saindrenan, P.; et al. Crosstalks between myo-inositol metabolism, programmed cell death and basal immunity in Arabidopsis. PLoS ONE 2009, 4, e7364. [CrossRef] [PubMed]

17. Luo, Y.; Qin, G.J.; Zhang, J.; Liang, Y.; Song, Y.Q.; Zhao, M.P.; Tsuge, T.; Aoyama, T.; Liu, J.J.; Gu, H.Y.; et al. D-myo-inositol-3-phosphate affects phosphatidylinositol-mediated endomembrane function in Arabidopsis and is essential for auxin-regulated embryogenesis. Plant Cell 2011, 23, 1352-1372. [CrossRef] [PubMed]

18. Donahue, J.L.; Alford, S.R.; Torabinejad, J.; Kerwin, R.E.; Nourbakhsh, A.; Ray, W.K.; Hernick, M.; Huang, X.; Lyons, B.M.; Hein, P.P.; et al. The Arabidopsis myo-inositol 1-phosphate synthase1 gene is required for myo-inositol synthesis and suppression of cell death. Plant Cell 2010, 22, 888-903. [CrossRef] [PubMed]

19. Raboy, V. Seeds for a better future: 'Low phytate' grains help to overcome malnutrition and reduce pollution. Trends Plant Sci. 2001, 6, 458-462. [CrossRef]

20. Baig, M.M.; Kelly, S.; Loewus, F. L-ascorbic acid biosynthesis in higher plants from L-gulono-1, 4-lactone and L-galactono-1, 4-lactone. Plant Physiol. 1970, 46, 277-280. [CrossRef] [PubMed]

21. Allison, J.H.; Stewart, M.A. Myo-inositol and ascorbic acid in developing rat brain. J. Neurochem. 1973, 20, 1785-1788. [CrossRef] [PubMed]

22. Banhegyi, G.; Braun, L.; Csala, M.; Puskás, F.; Mandl, J. Ascorbate metabolism and its regulation in animals. Free Radic. Biol. Med. 1997, 23, 793-803. [CrossRef]

23. Loewus, F. Inositol metabolism and cell wall formation in plants. Fed. Proc. 1965, 24, 855-862. [PubMed]

24. Loewus, F.A. Inositol and plant cell wall polysaccharide biogenesis. Subcell. Biochem. 2006, 39, 21-45. [PubMed]

25. Pang, C.Y.; Wang, H.; Pang, Y.; Xu, C.; Jiao, Y.; Qin, Y.M.; Western, T.L.; Yu, S.X.; Zhu, Y.X. Comparative proteomics indicates that biosynthesis of pectic precursors is important for cotton fiber and Arabidopsis root hair elongation. Mol. Cell Proteom. 2010, 9, 2019-2033. [CrossRef] [PubMed]

26. Li, H.B.; Qin, Y.M.; Pang, Y.; Song, W.Q.; Mei, W.Q.; Zhu, Y.X. A cotton ascorbate peroxidase is involved in hydrogen peroxide homeostasis during fibre cell development. New Phytol. 2007, 175, 462-471. [CrossRef] [PubMed]

27. Tao, C.C.; Jin, X.; Zhu, L.P.; Xie, Q.L.; Wang, X.C.; Li, H.B. Genome-wide investigation and expression profiling of APX gene family in Gossypium hirsutum provide new insights in redox homeostasis maintenance during different fiber development stages. Mol. Genet. Genom. 2018, 293, 685-697. [CrossRef] [PubMed]

28. Liu, G.J.; Xiao, G.H.; Liu, N.J.; Liu, D.; Chen, P.S.; Qin, Y.M.; Zhu, Y.X. Targeted Lipidomics Studies Reveal that Linolenic Acid Promotes Cotton Fiber Elongation by Activating Phosphatidylinositol and Phosphatidylinositol Monophosphate Biosynthesis. Mol. Plant 2015, 8, 911-921. [CrossRef] [PubMed]

29. Kusano, H.; Testerink, C.; Vermeer, J.E.; Tsuge, T.; Shimada, H.; Oka, A.; Munnik, T.; Aoyama, T. The Arabidopsis Phosphatidylinositol Phosphate 5-Kinase PIP5K3 is a key regulator of root hair tip growth. Plant Cell 2008, 20, 367-380. [CrossRef] [PubMed]

30. Stenzel, I.; Ischebeck, T.; König, S.; Hołubowska, A.; Sporysz, M.; Hause, B.; Heilmann, I. The type B phosphatidylinositol-4-phosphate 5-kinase 3 is essential for root hair formation in Arabidopsis thaliana. Plant Cell 2008, 20, 124-141. [CrossRef] [PubMed]

31. Abid, G.; Silue, S.; Muhovski, Y.; Jacquemin, J.M.; Toussaint, A.; Baudoin, J.P. Role of myo-inositol phosphate synthase and sucrose synthase genes in plant seed development. Gene 2009, 439, 1-10. [CrossRef] [PubMed]

32. Cui, M.; Liang, D.; Wu, S.; Ma, F.W.; Lei, Y.S. Isolation and developmental expression analysis of L-myo-inositol-1-1phosphate synthase in four Actinidia species. Plant Physiol. Biochem. 2013, 73, 351-358. [CrossRef] [PubMed]

33. Basak, P.; Maitra-Majee, S.; Das, J.K.; Mukherjee, A.; Ghosh Dastidar, S.; Pal Choudhury, P.; Lahiri Majumder, A. An evolutionary analysis identifies a conserved pentapeptide stretch containing 
the two essential lysine residues for rice L-myo-inositol 1-phosphate synthase catalytic activity. PLoS ONE 2017, 12, e0185351. [CrossRef] [PubMed]

34. Fleet, C.M.; Yen, J.Y.; Hill, E.A.; Gillaspy, G.E. Co-suppression of AtMIPS demonstrates cooperation of MIPS1, MIPS2 and MIPS3 in maintaining myo-inositol synthesis. Plant Mol. Biol. 2018, 97, 253-263. [CrossRef] [PubMed]

35. Nunes, A.C.; Vianna, G.R.; Cuneo, F.; Amaya-Farfán, J.; de Capdeville, G.; Rech, E.L.; Aragão, F.J. RNAi-mediated silencing of the myo-inositol-1-phosphate synthase gene (GmMIPS1) in transgenic soybean inhibited seed development and reduced phytate content. Planta 2006, 224, 125-132. [CrossRef] [PubMed]

36. Zhai, H.; Wang, F.B.; Si, Z.Z.; Huo, J.X.; Xing, L.; An, Y.Y.; He, S.Z.; Liu, Q.C. A myo-inositol-1-phosphate synthase gene, IbMIPS1, enhances salt and drought tolerance and stem nematode resistance in transgenic sweet potato. Plant Biotechnol. J. 2016, 14, 592-602. [CrossRef] [PubMed]

37. Tan, J.L.; Wang, C.Y.; Xiang, B.; Han, R.H.; Guo, Z.F. Hydrogen peroxide and nitric oxide mediated cold- and dehydration-induced myo-inositol phosphate synthase that confers multiple resistances to abiotic stresses. Plant Cell Environ. 2013, 36, 288-299. [CrossRef] [PubMed]

38. Latrasse, D.; Jégu, T.; Meng, P.H.; Mazubert, C.; Hudik, E.; Delarue, M.; Charon, C.; Crespi, M.; Hirt, H.; Raynaud, C.; et al. Dual function of MIPS1 as a metabolic enzyme and transcriptional regulator. Nucleic Acids Res. 2013, 41, 2907-2917. [CrossRef] [PubMed]

39. Valluru, R.; Van den Ende, W. Myo-inositol and beyond-emerging networks under stress. Plant Sci. 2011, 181, 387-400. [CrossRef] [PubMed]

40. Stevenson-Paulik, J.; Bastidas, R.J.; Chiou, S.T.; Frye, R.A.; York, J.D. Generation of phytate-free seeds in Arabidopsis through disruption of inositol polyphosphate kinases. Proc. Natl. Acad. Sci. USA 2005, 102, 12612-12617. [CrossRef] [PubMed]

41. Ischebeck, T.; Stenzel, I.; Heilmann, I. Type B phosphatidylinositol-4-phosphate 5-kinases mediate Arabidopsis and Nicotiana tabacum pollen tube growth by regulating apical pectin secretion. Plant Cell 2008, 20, 3312-3330. [CrossRef] [PubMed]

42. Kusuda, H.; Koga, W.; Kusano, M.; Oikawa, A.; Saito, K.; Hirai, M.Y.; Yoshida, K.T. Ectopic expression of myo-inositol 3-phosphate synthase induces a wide range of metabolic changes and confers salt tolerance in rice. Plant Sci. 2015, 232, 49-56. [CrossRef] [PubMed]

43. Zhang, M.; Zheng, J.Y.; Long, H.; Xiao, Y.H.; Yan, X.Y.; Pei, Y. Auxin regulates cotton fiber initiation via GhPIN-mediated auxin transport. Plant Cell Physiol. 2017, 58, 385-397. [CrossRef] [PubMed]

44. Samuel, Y.S.; Cheung, F.; Lee, J.J.; Ha, M.; Wei, N.E.; Sze, S.H.; Stelly, D.M.; Thaxton, P.; Triplett, B.; Town, C.D.; et al. Accumulation of genome-specific transcripts, transcription factors and phytohormonal regulators during early stages of fiber cell development in allotetraploid cotton. Plant J. 2006, 47, 761-765. [CrossRef] [PubMed]

45. Chen, H.; Xiong, L.M. myo-Inositol-1-phosphate synthase is required for polar auxin transport and organ development. J. Biol. Chem. 2010, 285, 24238-24247. [CrossRef] [PubMed]

46. Tamura, K.; Peterson, D.; Peterson, N.; Stecher, G.; Nei, M.; Kumar, S. MEGA5: Molecular evolutionary genetics analysis using maximum likelihood, evolutionary distance, and maximum parsimony methods. Mol. Biol. Evol. 2011, 28, 2731-2739. [CrossRef] [PubMed]

47. Jin, X.; Zhu, L.P.; Yao, Q.; Meng, X.R.; Ding, G.H.; Wang, D.; Xie, Q.L.; Tong, Z.; Tao, C.C.; Yu, L.; et al. Expression profiling of mitogen-activated protein kinase genes reveals their evolutionary and functional diversity in different rubber tree (Hevea brasiliensis) cultivars. Genes 2017, 8, 261. [CrossRef] [PubMed]

48. Zhu, L.P.; Jin, X.; Xie, Q.L.; Yao, Q.; Wang, X.C.; Li, H.B. Calcium-dependant protein kinase family genes involved in ethylene-induced natural rubber production in different Hevea brasiliensis cultivars. Int. J. Mol. Sci. 2018, 19, 947. [CrossRef] [PubMed]

49. Rozas, J.; Ferrer-Mata, A.; Sánchez-DelBarrio, J.C.; Guirao-Rico, S.; Librado, P.; Ramos-Onsins, S.E.; Sánchez-Gracia, A. DnaSP 6: DNA sequence polymorphism analysis of large datasets. Mol. Biol. Evol. 2017, 34, 3299-3302. [CrossRef] [PubMed]

50. Li, R.; Xin, S.; Tao, C.C.; Jin, X.; Li, H.B. Cotton ascorbate oxidase promotes cell growth in cultured tobacco bright yellow-2 cells through generation of apoplast oxidation. Int. J. Mol. Sci. 2017, 18, 1346. [CrossRef] [PubMed] 
(C) 2019 by the authors. Licensee MDPI, Basel, Switzerland. This article is an open access article distributed under the terms and conditions of the Creative Commons Attribution (CC BY) license (http://creativecommons.org/licenses/by/4.0/). 